\title{
Fuzzification of probabilistic objects
}

\author{
Martin Papčo * \\ Catholic University in Ružomberok, Hrabovská cesta 1, 03401 Ružomberok, Slovak Republic \\ Mathematical Institute, Slovak Academy of Sciences, Štefánikova 49, 81473 Bratislava, Slovak Republic \\ e-mail: papco@ruzomberok.sk
}

\begin{abstract}
A categorical approach to probability allows to put basic notions of probability into a broader mathematical perspective, to evaluate their roles, and mutual relationships. Classical probability theory and fuzzy probability theory lead to two particular categories and their relationship (in categorical terms) enable us to understand and explicitly formulate the difference between them. Using our previous results, we show that the category ID of D-posets of fuzzy sets provides a framework in which the transition from classical to fuzzy probability theory is the consequence of some natural assumptions imposed on classical notions. Probability domains are constructed via suitable cogenerators and we study the transition in terms of the fuzzification of classical Boolean cogenerator. We introduce two categories CP and FP of probability spaces and observables corresponding to the classical probability theory and the fuzzy probability theory, respectively. We show that CP is isomorphic to a subcategory of FP.
\end{abstract}

Keywords: domain of probability, cogenerator, classical probability theory, fuzzy probability theory, D-poset, sub-D-poset, fuzzification, category of D-posets of fuzzy sets, divisible D-poset, effect algebra, observable, probability measure, probability integral, MV-algebra, bold algebra, Łukasiewicz tribe, classical probability category, fuzzy probability category

\section{Introduction}

In the classical probability theory, CPT for short ([18], [20]), random events are modelled by fields of sets having the following additional property: in order to capture limit stochastics, random events are supposed to be closed with respect to sequential limits, i.e., we assume that random events form a $\sigma$-field of sets.

In the so-called fuzzy probability theory, FTP for short ([16]), also known as operational probability theory $([1],[2])$, random events are modelled by suitable systems of fuzzy sets. This generalization of classical random events allows to model some quantum phenomena. Namely, a fuzzy random variable

*This work was supported by the Slovak Research and Development Agency [contract No. APVV-0178-11]; and Slovak Scientific Grant Agency [VEGA project 2/0046/11]. can send an outcome of a random experiment into a genuine probability measure. Further, the transition from classical random events to fuzzy random events corresponds to the ideas of L.A. Zadeh, who has proposed in [27] to fuzzify CPT via replacing a $\sigma$-field $\mathbb{A}$ of subsets of $\Omega$ by the system $\mathcal{M}(\mathbb{A})$ of all measurable functions ranging in the closed unit interval $[0,1]$, and replacing a probability measure $P$ by the probability integral $\int u \mathrm{~d} P$, $u \in \mathcal{M}(\mathbb{A})$. Further he proposed to generalize the Boolean operations on random events to coordinatewise $\vee$ and $\wedge$ operations: for $u, v \in \mathcal{M}(\mathbb{A})$, we put $(u \vee v)(\omega)=u(\omega) \vee v(\omega),(u \wedge v)(\omega)=u(\omega) \wedge v(\omega)$, $\omega \in \Omega$, and for $u \in \mathcal{M}(\mathbb{A})$ we define its complement $u^{c}$ as $u^{c}(\omega)=1-u(\omega), \omega \in \Omega$. Also, FPT allows to generalize important constructions of CPT such as conditional probability, conditional expectations, independence of fuzzy events (effects) and observables (dual maps to fuzzy random variables), and Markov processes (see [16], [17]).

As it is known ([21], [26], [5]), there are other canonical ways how to generalize the Boolean operations in order to develop a reasonable fuzzy stochastics. In FPT and some other generalizations of probability theory, effect algebras $([6])$ equivalently D-posets ([19], [4]) — and MV-algebras model the algebraic part of the theories. Valuable comments on generalizations of probability theory can be found in [22].

Using our previous results (cf. [23], [24], [25], [9], [10], [11], [12], [13]), we show that the category of Dposets of fuzzy sets provides a framework in which the transition from CPT to FPT is the consequence of some natural assumptions imposed on CPT.

A categorical approach to probability allows to put all basic notions into a broader mathematical perspective, to evaluate their roles, and mutual relationships ([14]). CPT and FPT lead to two particular categories and their relationship (in categorical terms) enable us to understand and explicitly formulate the difference between CPT and FPT.

In the next section we recall the construction of domains of probability via a cogenerator ([9], [10]).

\section{Domains of probability}

Elementary events, random events, probability measures, random variables, and observables constitute central notions of the probability theory language. Each of them captures some reality cut-out 
connected with specific and complex type of uncertainty. In a relaxed way the situation in question can be viewed as some sort of drama. Elementary events (random experiment outcomes) are its characters (properties), random events describe relationships among characters (fellowships), probability measures state their power (importance), random variables enable to tell a story in the costumes of real numbers (measurements) and, finally, observables provide effective tools to compare two dramas. CPT can be characterized by all features mentioned above. The problem is that the resulting theory controls only a limited piece of reality, namely the black-and-white one, described by yesno questions-answers. From this point of view, FPT captures wider spectrum of situations: we allow questions with more than two opposite answers (e.g. yes, no, unknown).

A generalized pipeline introduced in [12] serves as an illustrating example of such "fuzzy" stochastic situation. It deals with a redistribution of a unit volume of liquid and, from the mathematical viewpoint, it models a transformation of one discrete probability space into another one. Simple pipelines (represented by classical maps) do not allow to solve all situations, but multivalued (fuzzy) pipelines provide solutions for every pair of discrete probability spaces. This leads to the notion of a "fuzzy" (operational) random variable and yields an alternative approach to random walks (cf. [13]).

A categorical approach to probability enables us to incorporate both, CPT and FPT, into a generalized probability theory, GPT for short, in which they become special cases. In GPT, random events are represented by objects of a suitable chosen category, and the other three CPT basic notions are defined in terms of morphisms and, moreover, simple categorical constructions correspond to important probabilistic notions and guarantee their properties.

In this section, we deal with a categorical approach to domains of probability. Based on previous results (cf. [9]), our procedure can be summarized as follows (see Fig. 1):

- Start with a "system $\mathcal{A}$ of events";

- Choose an "evaluator $C$ "- a cogenerator; usually a structured set suitable for "evaluating" (e.g. the two element Boolean algebra, unit interval carrying Łukasiewicz MV-structure, Dposet, ...);

- Choose a set $X$ of "properties" evaluated via $C$ such that $X$ separates $\mathcal{A}$;

- Represent each event $a \in \mathcal{A}$ via the "evaluation" of $\mathcal{A}$ into $C^{X}$ assigning each $a \in \mathcal{A}$ its evaluation $a_{X} \in C^{X}, a_{X} \equiv\{x(a) ; x \in X\}$;

- Form the minimal "subalgebra" $D$ of $C^{X}$ containing $\left\{a_{X} ; a \in \mathcal{A}\right\}$ (see Fig. 2);

- The subalgebra forms a probability domain $D \subseteq C^{X}$.

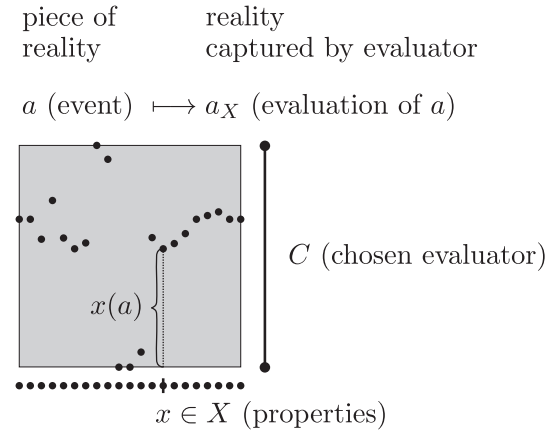

Figure 1: Representation of an event.

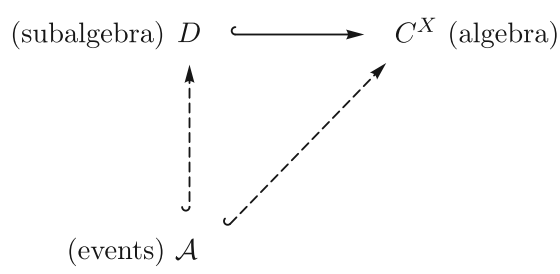

Figure 2: Subalgebra of events.

In the approach described above, the cogenerator carrying a suitable structure to evaluate events plays a crucial role. The two-element Boolean algebra $\{0,1\}$ as the cogenerator $C$ yields some $\sigma$-algebra of random events as the domain of probability in CPT (each subset of universe can be represented via its indicator function). For a change, the unit interval $[0,1]$ equipped by the Eukasiewicz $\mathrm{MV}$-structure as the cogenerator $C$ produces some system of fuzzy sets which can serve as the probability domain in FPT.

In general, each choice of a suitable cogenerator $C$ leads to the domain of probability of some particular probability theory and such domains can be described as objects of a suitable (sub)category. Because there are more than one probability theory, it is desirable to define a reference category and its subcategories corresponding to particular probability theories and some functors between these subcategories corresponding to the relationships between the probability theories in question. Referring to our previous results in [9] and [10], we claim that the unit interval $I=[0,1]$ considered as a D-poset is the cogenerator which yields a suitable reference category, namely the category ID of D-posets of fuzzy sets and sequentially continuous D-homomorphisms.

$D$-posets have been introduced by F. Kôpka and F. Chovanec in [19] in order to model quantum phenomena. They generalize ([4]) various structures, e.g. D-lattices, orthoalgebras, Boolean algebras, $\mathrm{MV}$-algebras and provide a category in which states and observables become morphisms [3]. Recall that a $D$-poset is a partially order set $X$ with the least element $0_{X}$, the greatest element $1_{X}$, and a partial binary operation called difference, such that $a \ominus b$ is defined iff $b \leq a$, and the following axioms are assumed: 
(D1) $a \ominus 0_{X}=a$ for every $a \in X$;

(D2) If $c \leq b \leq a$, then $a \ominus b \leq a \ominus c$ and $(a \ominus c) \ominus$ $(a \ominus b)=b \ominus c$.

Recall that each D-poset can be reorganized into an effect algebra and the two structures are isomorphic (cf. [5], [25]).

Essential to probabilistic applications ([10], [13], [12], [24]) are D-posets of fuzzy sets, i.e. system $\mathcal{X} \subseteq I^{X}$ equipped with the coordinatewise partial order, coordinatewise convergence of sequences, the bottom and the top element of $I^{X}$, and closed with respect to the partial operation difference defined coordinatewise. If for each $x, y \in X, x \neq y$, there exists $u \in \mathcal{X}$ such that $u(x) \neq u(x)$, then $\mathcal{X}$ is said to be reduced. We always assume that $\mathcal{X}$ is reduced. Denote ID the category having (reduced) D-posets of fuzzy sets as objects and having sequentially continuous D-homomorphisms as morphisms. Objects of ID are subobjects of the powers $I^{X}$ (cf. [23]).

An ID-poset allows us to model the sure and impossible events, and the negation of an event as the difference of the sure event and the event in question. It is an efficient simple structure in which random events in both CPT and FPT can be described and their structure is completely determined by the morphisms into cogenerator $C=[0,1]$. Thus it makes sense to study probability domains within the category ID.

We identify each subset $A$ of a universe $X$ and its indicator function $\chi_{A}, \chi_{A}(x)=1$ if $x \in A$ and $\chi_{A}(x)=0$ otherwise. Then ([23]) a $\sigma$-field $\mathbb{A}$ of subsets of $X$ can be viewed as an ID-poset having some additional properties, where $\chi_{A} \ominus \chi_{B}=\chi_{A}-\chi_{B}$ is defined iff $B \subseteq A$. Similarly, the measurable functions $\mathcal{M}(\mathbb{A})$ can be viewed as an ID-poset having some additional properties, where for $u, v \in \mathcal{M}(\mathbb{A})$ we define $u \ominus v=u-v$ iff $v(x) \leq u(x)$ for all $x \in X$. Now, let $\mathbb{B}$ be a $\sigma$-field of subsets of $Y$ and let $f: X \rightarrow Y$ be a measurable map. Then the preimage map $f^{\leftarrow}: \mathbb{B} \rightarrow \mathbb{A}, f^{\leftarrow}(B)=\{x \in X$; $f(x) \in B\}$, is a sequentially continuous Boolean homomorphism of $\mathbb{B}$ into $\mathbb{A}$. It is easy to see that every probabilistic integral (defined for [0,1]-valued functions measurable with respect to a probability measure) and every probability measure (as the integral reduced to indicator functions) are sequentially continuous D-homomorphisms (the sequential continuity follows from the Lebesgue dominated convergence theorem). More important is that also the converse holds true (cf. [7]).

Lemma 2.1. (i) Let $p$ be a sequentially continuous $D$-homomorphism of $\mathbb{A}$ into $I$. Then $p$ is a probability measure.

(ii) Let $h$ be a sequentially continuous $D$ homomorphism of $\mathcal{M}(\mathbb{A})$ into $I$. Then there exists a probability measure $P$ on $\mathbb{A}$ such that for each $u \in \mathcal{M}(\mathbb{A})$ we have $h(u)=\int u \mathrm{~d} P$, i.e., $h$ is a probability integral on $\mathcal{M}(\mathbb{A})$ with respect to $P$.
Consequently observables, probability measures, and fuzzy probability measures become morphisms of the category ID ([11]).

Observe that nontraditional cogenerators lead to nontraditional models of probability. For example, for a given positive integer $n$, the cogenerator $S_{n}=\left\{\left(x_{1}, x_{2}, \ldots, x_{n}\right) \in I ; \sum_{i=1}^{n} x_{i} \leq 1\right\}$ yields a generalized fuzzy probability, in which each generalized random event represents $n$ "competing subjects" ([8]). More information on other nonclassical cogenerators and the corresponding (probability) theories can be found in [10], [12], [13], [15].

\section{Fuzzification of a cogenerator}

In this section we formalize the transition from $\{0,1\}$ (Boolean cogenerator) to $I$ (fuzzy cogenerator).

Let $A$ be a D-poset. By a sub-D-poset of $A$ we understand a subset $B$ of $A$ containing the top and the bottom element of $A$, equipped with the inherited partial order and closed with respect to the inherited partial operation of difference.

Recall ([5], [7]) that a bold algebra is a system $\mathcal{X} \subseteq[0,1]^{X}$ containing the constant functions $0_{X}$, $1_{X}$ and closed with respect to the complement and (Eukasiewicz) operations $\oplus, \odot$ : for $a, b \in \mathcal{X}$ put $(a \oplus b)(x)=a(x) \oplus b(x)=\min \{1, a(x)+b(x)\}$, $(a \odot b)(x)=a(x) \odot b(x)=\max \{0, a(x)+b(x)-1\}$, $x \in X$. Bold algebras are MV-algebras representable as $[0,1]$-valued functions. Also the unit interval $I=[0,1]$ can be considered as a bold algebra of all measurable $[0,1]$-valued functions. Indeed, let $\mathbb{T}$ be the trivial field of all subsets of a singleton $\{\cdot\}$. Then $[0,1] \equiv[0,1]^{\{\cdot\}}=\mathcal{M}(\mathbb{T})$. If a bold algebra $\mathcal{X} \subseteq[0,1]^{X}$ is sequentially closed in $[0,1]^{X}$, then $\mathcal{X}$ is a Eukasiewicz tribe.

Lemma 3.1. Let $B$ be a sub-D-poset of $I$ and let $a, b \in B$. Then

(i) $\{1-[(1-a) \wedge b]\} \geq a$;

(ii) $1-(\{1-[(1-a) \wedge b]\}-a)=\min \{1, a+b\}$.

Proof. If $(1-a) \wedge b=1-a$, then $1 \leq a+b$, thus $a=$ $\{1-[(1-a) \wedge b]\} \geq a$ and $1-(\{1-[(1-a) \wedge b]\}-a)$ $=1$.

If $(1-a) \wedge b=b$, then $1 \geq a+b$, thus $1-b=$ $\{1-[(1-a) \wedge b]\} \geq a$ and $1-(\{1-[(1-a) \wedge b]\}-a)$ $=a+b$.

Corollary 3.2. Let $B$ be a sub-D-poset of $I$. Then $B$ is closed with respect to the Eukasiewicz operations $a \oplus b=\min \{1, a+b\}, a \odot b=\max \{0, a+b-1\}$.

Lemma 3.3. Let $B$ be a bold subalgebra of $I$. For $a, b \in B, b \leq a$, put $a \ominus b=a-b$. Then $B$ equipped with the inherited order and the difference " $\ominus$ " is a sub-D-poset of $I$.

Corollary 3.4. There is a canonical one-to-one relationship between sub-D-posets of I and bold subalgebras of $I$. 
It is easy see that a subtractivity of a Dhomomorphisms into $I$ is equivalent to the additivity of states (see [11]).

Definition 3.5. Let $A$ be a D-poset and let $n$ be a natural number, $n>1$. Assume that for each $a \in A, a \neq 0$, there exists an element $a(n) \in A$ such that $0<a(n)<a$ and, for each $k=1,2, \ldots, n-1$, we can subtract from $a$ successively $k$-times $a(n)$ and the result is greater or equal to $a(n)$, and if we subtract from $a$ successively $n$-times $a(n)$, then the result is 0 . Then $A$ is said to be divisible by $n$. If $A$ is divisible by $n$ for each natural number $n, n>1$, then $A$ is said to be divisible.

Definition 3.6. Let $A$ be a D-poset and let $B$ be a sub-D-poset of $A$. Assume that for each countable set $S \subset B$ there exists the supremum $\sup S$ of $S$ in $A$ and $\sup S \in B$. Then $B$ is said to be $\sigma$-complete in $A$.

Lemma 3.7. Let $B$ be a sub-D-poset of $I$. If $B$ is divisible and $\sigma$-complete in $I$, then $B=I$.

Corollary 3.8. I is the smallest of all sub-Dposets $B$ of $I$ such that $B$ is divisible and $\sigma$-complete in $I$.

This yields a description of the relationship between $\{0,1\}$ and $[0,1]$ and leads to the following definition.

Definition 3.9. Let $A$ be a $\sigma$-complete divisible D-poset and let $B$ be a sub-D-poset of $A$. If there is no proper sub-D-poset $C$ of $A$ such that

(i) $B$ is a sub-D-poset of $C$,

(ii) $C$ is divisible,

(iii) $C$ is $\sigma$-complete in $A$,

then $A$ is said to be a fuzzification of $B$.

\section{Fuzzification of objects}

First, we show that $\mathcal{M}(\mathbb{A})$ is a fuzzification of $\mathbb{A}$.

Let $\mathbb{A}$ be a (reduced) $\sigma$-field of subsets of a set $X$ and let $\mathcal{M}(\mathbb{A})$ be the set of all measurable functions ranging in $[0,1]$. Recall that both $\mathbb{A}$ and $\mathcal{M}(\mathbb{A})$ are Eukasiewicz tribes and D-posets of fuzzy sets with respect to the coordinatewise partial order and the inherited difference operation defined coordinatewise.

Lemma 4.1. The sub-D-poset $\mathcal{M}(\mathbb{A})$ is divisible and $\sigma$-complete in $[0,1]^{X}$.

Proof. Clearly, $\mathcal{M}(\mathbb{A})$ is divisible. Let $\left\{u_{n}\right\}_{n=1}^{\infty}$ be a sequence of elements of $\mathcal{M}(\mathbb{A})$. For $n=1,2, \ldots$, put $v_{n}=\bigvee_{k=1}^{n} u_{k}\left(v_{n}(x)=\sup \left\{u_{1}(x), u_{2}(x), \ldots, u_{n}(x)\right\}\right.$, $x \in X)$. Then $\left\{v_{n}\right\}_{n=1}^{\infty}$ is a nondecreasing sequence converging in $[0,1]^{X}$ to $v=\sup \left\{u_{1}, u_{2}, \ldots\right\}$. Since the sub-D-poset $\mathcal{M}(\mathbb{A})$ is closed with respect to limits, we have $v \in \mathcal{M}(\mathbb{A})$. Thus $\mathcal{M}(\mathbb{A})$ is $\sigma$-complete in $[0,1]^{X}$.
Lemma 4.2. Let $\mathcal{X}$ be a sub-D-poset of $[0,1]^{X}$ such that $\mathbb{A} \subseteq \mathcal{X}$. If $\mathcal{X}$ is divisible and $\sigma$-complete in $[0,1]^{X}$, then $\mathcal{M}(\mathbb{A}) \subseteq \mathcal{X}$ and the sub-D-poset $\mathcal{M}(\mathbb{A})$ is $\sigma$-complete in $\mathcal{X}$.

Proof. Since $\mathcal{X}$ is $\sigma$-complete in $[0,1]^{X}$, for $u, v \in \mathcal{X}$ we have $u \vee v \in \mathcal{X}$. It is known (cf. [10]) that each lattice D-poset of fuzzy sets is a bold algebra. Thus $\mathcal{X}$ is a bold algebra. Since $\mathcal{X}$ is $\sigma$-complete in $[0,1]^{X}$, it follows that $\mathcal{X}$ is sequentially closed in $[0,1]^{X}$, and hence $\mathcal{X}$ is a Eukasiewicz tribe. It is known (cf. [10]) that for each divisible Łukasiewicz tribe $\mathcal{X} \subseteq[0,1]^{X}$ there exists a $\sigma$-field $\mathbb{B}$ of subsets of $X$ such that $\mathcal{X}=\mathcal{M}(\mathbb{B})$. From $\mathbb{A} \subseteq \mathcal{X}$ we get $\mathcal{M}(\mathbb{A}) \subseteq \mathcal{X}$

Corollary 4.3. $\mathcal{M}(\mathbb{A})$ is the smallest of all sub-Dposets $\mathcal{X}$ of $[0,1]^{X}$ such that $\mathbb{A}$ is contained in $\mathcal{X}$ and $\mathcal{X}$ is divisible and $\sigma$-complete in $[0,1]^{X}$.

Now, let us turn to categorical aspects of the transition from classical probability to fuzzy probability.

Definition 4.4. Let $(\Omega, \mathbb{A}, P)$ and $(\Xi, \mathbb{B}, Q)$ be classical probability spaces and let $h$ be a sequentially continuous D-homomorphisms of $\mathbb{B}$ into $\mathbb{A}$ such that $Q(B)=P(h(B))$ for all $B \in \mathbb{B}$. Then $h$ is said to be measure preserving. It will be called a classical observable.

Definition 4.5. Let $(\Omega, \mathbb{A}, P)$ be a classical probability space, let $\mathcal{M}(\mathbb{A})$ be the corresponding $\mathrm{D}$-poset of fuzzy sets and let $\int(\cdot) \mathrm{d} P$ be the probability integral with respect to $P$. Then $\left(\Omega, \mathcal{M}(\mathbb{A}), \int(\cdot) \mathrm{d} P\right)$ is said to be a fuzzy probability space.

Observe that there is a canonical one-to-one correspondence between classical probability spaces and fuzzy probability spaces.

Definition 4.6. Let $\left(\Omega, \mathcal{M}(\mathbb{A}), \int(\cdot) \mathrm{d} P\right)$ and $\left(\Xi, \mathcal{M}(\mathbb{B}), \int(\cdot) \mathrm{d} Q\right)$ be fuzzy probability spaces. Let $h$ be a sequentially continuous D-homomorphisms of $\mathcal{M}(\mathbb{B})$ into $\mathcal{M}(\mathbb{A})$ such that $\int v \mathrm{~d} Q=\int h(v) \mathrm{d} P$ for each $v \in \mathcal{M}(\mathbb{B})$. Then $h$ is said to be probability integral preserving. It will be called a fuzzy observable. If, moreover, $h(B) \in \mathbb{A}$ for all $B \in \mathbb{B}$, then $h$ is said to be a restricted fuzzy observable.

It is known (cf. [1], [2], [16], [11], [12]) that there are fuzzy observables which are not restricted. Such observables correspond to genuine fuzzy (operational) random variables and have definite quantum qualities.

Denote CP the category having classical probability spaces as objects and classical observables as morphisms. Denote FP the category having fuzzy probability spaces as objects and fuzzy observables as morphisms.

QUESTION. How are the categories CP and FP related?

To answer our question, we recall the following extension theorem (cf. [7], [11]). 
Theorem 4.7. Let $(\Omega, \mathbb{A}, P)$ and $(\Xi, \mathbb{B}, Q)$ be classical probability spaces and let $\left(\Omega, \mathcal{M}(\mathbb{A}), \int(\cdot) \mathrm{d} P\right)$ and $\left(\Xi, \mathcal{M}(\mathbb{B}), \int(\cdot) \mathrm{d} Q\right)$ be the corresponding fuzzy probability spaces. Let $h_{c}$ be a classical observable. Then there exists a unique fuzzy observable $h$ such that $h_{c}(B)=h(B)$ for all $B \in \mathbb{B}$.

Denote RFP the subcategory of FP having the fuzzy probability spaces as objects and the restricted fuzzy observables as morphisms.

The next theorem follows directly from the previous one.

Theorem 4.8. The categories $C P$ and $R F P$ are isomorphic.

ANSWER. There is a canonical isomorphism between $\mathrm{CP}$, representing the classical probability theory, and the subcategory RFP of FP, representing the fuzzy probability theory. The objects of the two categories are in a canonical one-to-one correspondence, but the fuzzy probability theory has "more" morphisms. Indeed, to each classical domain of probability $\mathbb{A}(\sigma$-field of sets) there corresponds a unique domain of fuzzy probability theory $\mathcal{M}(\mathbb{A})$ (the set of all measurable functions ranging in $I$ ) but, in general, there are fuzzy observables of $\mathcal{M}(\mathbb{B})$ to $\mathcal{M}(\mathbb{A})$ which are not extensions of any classical random variable from $\mathbb{B}$ to $\mathbb{A}$.

The author is indebted to the referee for his valuable suggestions how to improve the original text.

\section{References}

[1] S. Bugajski. Statistical maps I. basic properties. Math. Slovaca, 51:321-342, 2001.

[2] S. Bugajski. Statistical maps II. operational random variables. Math. Slovaca, 51:343-361, 2001.

[3] F. Chovanec and R. Frič. States as morphisms. Internat. J. Theoret. Phys., 49:30503060, 2010.

[4] F. Chovanec and F. Kôpka. D-posets. In D. M. Gabbay K. Engesser and D. Lehmann, editors, Handbook of Quantum Logic and Quantum Structures: Quantum Structures, pages 367-428, Amsterdam, 2007. Elsevier.

[5] A. Dvurečenskij and S. Pulmannová. New Trends in Quantum Structures. Kluwer Academic Publ. and Ister Science, Dordrecht and Bratislava, 2000.

[6] D.J. Foulis and M.K. Bennett. Effect algebras and unsharp quantum logics. Found. Phys., 24:1331-1352, 1994.

[7] R. Frič. Extension of domains of states. Soft Comput., 13:63-70, 2009.
[8] R. Frič. Simplex-valued probability. Math. Slovaca, 60:607-614, 2010.

[9] R. Frič and M. Papčo. On probability domains. Internat. J. Theoret. Phys., 49:30923100, 2010.

[10] R. Frič and M. Papčo. On probability domains II. Internat. J. Theoret. Phys., 50:37783786, 2011.

[11] R. Frič and M. Papčo. A categorical approach to probability theory. Studia Logica, 94:215230, 2010.

[12] R. Frič and M. Papčo. Fuzzification of crisp domains. Kybernetika, 46:1009-1024, 2010.

[13] R. Frič and M. Papčo. Statistical maps and generalized random walks. Math. Slovaca, 62:1079-1090, 2012.

[14] J.A. Goguen. A categorical manifesto. Math. Struct. in Comp. Science, 1:49-67, 1991.

[15] J.A. Goguen. L-fuzzy sets. J. Math. Anal. Appl., 18:145-174, 1967.

[16] S. Gudder. Fuzzy probability theory. Demonstratio Math., 31:235-254, 1998.

[17] S. Gudder. Observables and statistical maps. Foundations of Physics, 29:877-897, 1999.

[18] A.N. Kolmogorov. Grundbegriffe der Wahrscheinlichkeitsrechnung. Springer, Berlin, 1933.

[19] F. Kôpka and F. Chovanec. D-posets. Math. Slovaca, 44:21-34, 1994.

[20] M. Loève. Probability theory. D. Van Nostrand, Inc., Princeton, New Jersey, 1963.

[21] R. Mesiar. Fuzzy sets and probability theory. Tatra Mountains Mathematical Publ., 1:105123, 1992.

[22] M. Navara. Probability theory of fuzzy events. In P. Sobrevilla E. Montseny, editor, Fourth Conference of the European Society for Fuzzy Logic and Technology and 11 Rencontres Francophones sur la Logique Floue et ses Applications, pages 325-329, Barcelona, 2005. Universitat Politecnica de Catalunya.

[23] M. Papčo. On measurable spaces and measurable maps. Tatra Mountains Mathematical Publ., 28:125-140, 2004.

[24] M. Papčo. On fuzzy random variables: examples and generalizations. Tatra Mountains Mathematical Publ., 30:175-185, 2005.

[25] M. Papčo. On effect algebras. Soft Comput., 12:373-379, 2008.

[26] B. Riečan and D. Mundici. Probability on MValgebras. In E. Pap, editor, Handbook of Measure Theory, volume II, pages 869-910, Amsterdam, 2002. North-Holland.

[27] L.A. Zadeh. Probability measures of fuzzy events. J. Math. Anal. Appl., 23:421-427, 1968. 